\title{
Trastornos de sueño asociados a bruxismo de sueño en niños entre 3 y 6 años de edad atendidos en la Clínica Odontológica de la Universidad Mayor de Santiago, Chile
}

\section{Sleep disorders associated with sleep bruxism in children between 3 and 6 years old attended at the Dental Clinic of the University Mayor of Santiago, Chile}

Köstner Uribe S*, Brunet Echavarría J**, Tapia Vargas A***

\section{RESUMEN}

El bruxismo se define como una actividad repetitiva de los músculos masticatorios caracterizada por el apriete o rechinamiento dentario, y/o la tensión o desplazamiento de la mandíbula. ${ }^{1}$ Éste tiene dos manifestaciones circadianas: Puede ocurrir durante el sueño (BS) o durante vigilia. ${ }^{2}$ En niños, el método clínico más confiable para diagnosticarlo sigue siendo el basado en el reporte de rechinamiento por parte de sus padres o cuidadores; sin embargo, la mayoría de los niños duermen lejos de los padres. ${ }^{3}$ Existe una dificultad diagnóstica para poder establecer la pérdida de estructura dentaria por bruxismo en niños, ya que se debe diferenciar del desgaste fisiológico que se produce en los dientes temporales. Hay una serie de trastornos de sueño, catalogados por la Clasificación Internacional de Trastornos del Sueño ${ }^{4}$ que se han asociado al BS. 5, 6,7 El objetivo de este estudio fue establecer si existe asociación entre BS y determinados trastornos de sueño en preescolares. Se evaluaron 100 niños de la Clínica Odontológica de la Universidad Mayor de Santiago, Chile, de edades entre 3 y 6 años, mediante evaluación clínica y anamnesis consultada a padres o tutores para determinar la presencia de BS, además de encuesta "Escala de Alteraciones del Sueño en la Infancia, para preescolares". El estudio mostró una prevalencia de BS de un 47\%, sin diferencia entre hombres y mujeres y una asociación de algunos de los trastornos del sueño con BS (Parasomnias, Problemas Respiratorios, Hiperhidrosis y Sueño no reparador). Como conclusión sí existe asociación entre trastornos de sueño y BS.

PALABRAS CLAVE: Bruxismo, bruxismo de sueño, trastornos de sueño, niños, preescolares.

\section{SUMMARY}

Bruxism is defined as a repetitive activity of the chewing muscles characterized by grinding and clenching the teeth, and / or tension or displacement of the jaw ${ }^{20}$. This one has two circadian manifestations: It can occur during sleep

* Cirujano-Dentista. Facultad de Ciencias. Universidad Mayor. Docente asociada Asignatura Oclusión, Universidad Mayor.

* * Cirujano-Dentista. Docente asociada Asignatura Oclusión y Departamento de Odontología Integral del Adulto. Facultad de Ciencias. Universidad Mayor.

*** Cirujano-Dentista. Docente asociado. Asignatura Oclusión y Departamento de Odontología Integral del Adulto. Facultad de Ciencias. Universidad Mayor. 
(BS) or during wakefulness ${ }^{12}$. In children, the most reliable clinical method to diagnose is still based on the report of grinding by their parents or caregivers; however, most children sleep away from parents ${ }^{10}$. There is a diagnostic difficulty in establishing the loss of tooth structure due to bruxism in children, since it must be differentiated from the physiological wear that occurs in the temporary teeth. There are some sleep disorders, cataloged by the International Classification of Sleep Disorders ${ }^{41}$ that have been associated with BS ${ }^{6,17,31}$. The objective of this study was to establish whether there is an association between BS and sleep disorders in preschoolers. 100 children in the Odontological Clinic of the Universidad Mayor of Santiago, Chile, between 3 and 6 years old were evaluated, through clinical evaluation and anamnesis consulted to parents or guardians to determine the presence of BS, in addition to the survey "Scale of Sleep Disorder in Childhood, for preschoolers. " The study showed a prevalence of BS of 47\%, with no difference between boys and girls, and an association of some of the sleep disorders with BS (Parasomnias, Respiratory Problems, Hyperhidrosis and Not Restful Sleep). As a conclusion, there is an association between sleep disorders and BS.

KEY WORDS: Bruxism, sleep bruxism, sleep disorders, children, preschool.

Fecha de recepción: 3 de abril de 2019

Fecha de aceptación: 10 de abril de 2019.

Köstner Uribe S, Brunet Echavarría J, Tapia Vargas A. Trastornos de sueño asociados a bruxismo de sueño en niños entre 3 y 6 años de edad atendidos en la Clínica Odontológica de la Universidad Mayor de Santiago, Chile. 2019; 35, (2): 83-91

\section{INTRODUCCIÓN}

Según la última definición consensuada internacionalmente el 2013, el bruxismo se define como una actividad repetitiva de los músculos masticatorios caracterizada por el apriete o rechinamiento dentario, y/o la tensión o desplazamiento de la mandíbula.1 El bruxismo tiene dos manifestaciones circadianas: Puede ocurrir durante el sueño, indicado como bruxismo del sueño (BS de ahora en adelante) o durante la vigilia, indicado como bruxismo de vigilia. ${ }^{2}$

El bruxismo en general se clasifica como primario o idiopático, cuando no se reconocen problemas o causas médicas; y secundario o iatrogénico, cuando se asocia a problemas de tipo neurológico, psiquiátrico, administración de drogas, algunos medicamentos y trastornos de sueño. ${ }^{8}$

Las manifestaciones motoras orales de este trastorno son el apriete y rechinamiento dentario, durante el sueño o vigilia, ${ }^{3}$ que se pueden manifestar en signos y síntomas clínicos observables tales como pérdida de estructura dentaria, hipersensibilidad pulpar, dolor muscular, desórdenes temporomandibulares y cefalea. $^{9}$

Estas manifestaciones orales se presentan con fre- cuencia en adultos, pero también pueden afectar a niños, constituyendo un cuadro de etiología multifactorial, ${ }^{10}$ asociado a factores oclusales, psicosociales y ambientales, así como también a estados de estrés y/o ansiedad del niño. Sin embargo, existe una dificultad diagnóstica para poder establecer la pérdida de estructura dentaria por bruxismo en niños, porque se utilizan diferentes metodologías y también se debe diferenciar del desgaste fisiológico que se produce en los dientes temporales, lo que dificulta establecer su prevalencia. ${ }^{11}$ Este trastorno presenta una mayor prevalencia en niños de 3 a 5 años, disminuyendo considerablemente con la edad y durante la adolescencia. ${ }^{5}$

Actualmente, existen tres niveles de clasificación diagnóstica de bruxismo. En primer lugar, se habla de bruxismo "posible" (tanto de sueño como de vigilia), cuando se realiza un autoreporte a través de una encuesta y/o la anamnesis. En segundo lugar, se habla de bruxismo "probable" (de sueño o vigilia), basado en el autoreporte sumado a la inspección a través de la examinación clínica. El diagnóstico "definitivo" de BS, se basa en el autoreporte, la examinación clínica y además, en la polisomnografía (audio/video), a diferencia del diagnostico "definitivo" de bruxismo de vigilia, donde se debe realizar el auto reporte a través del cuestionario, examinación clínica y la grabación de la electromiografía. La poli- 
somnografía es el gold standard para el diagnóstico de BS. ${ }^{1}$ Sin embargo, tiene varias desventajas, ya que es un procedimiento intrahospitalario, de alto costo, tiene poca disponibilidad en centros médicos o no está disponible para pacientes pediátricos, requiere largo tiempo de realización (procedimiento se realiza durante toda la noche) y requiere tiempo de adaptación por parte del paciente..$^{12,13}$

En niños, el método clínico más confiable para diagnosticar bruxismo sigue siendo el basado en el reporte de rechinamiento por parte de padres o cuidadores; sin embargo, existe la limitación de que la mayoría de los niños duermen separados de los padres, quienes, por lo tanto, no son siempre conscientes del bruxismo de sus hijos. ${ }^{3}$

El desgaste patológico es difícil de cuantificar y de determinar, pero se define como aquel desgaste de los dientes que es atípico para la edad del paciente (exposición dentinaria), causando dolor o malestar, problemas funcionales, o deterioro de la apariencia estética, y que, si progresa, puede dar lugar a complicaciones indeseables de creciente complejidad. ${ }^{14}$

El bruxismo puede generar un impacto negativo en la calidad de vida y también es considerado un factor de riesgo de trastornos temporomandibulares (TTM), en la adolescencia y adultez. Puede generar desgaste dentario, y en casos más severos, trauma dentario. ${ }^{11}$

Entre los posibles factores patofisiológicos relacionados con la presencia de bruxismo en niños, existe una serie de trastornos de sueño, catalogados por la Clasificación Internacional de Trastornos del Sueño $(\mathrm{CITS})^{4}$ que se han asociado al BS, , 6, 7 entre ellos, parasomnias, trastornos en el inicio y mantenimiento de sueño, problemas respiratorios, excesiva somnolencia, hiperhidrosis de sueño, y sueño no reparador.

La Asociación Americana de Medicina del Sueño (AAMS), el año 2016, realizó un consenso acerca del número de horas diarias que deben dormir los niños: los menores entre 3 y 5 años deben dormir entre diez $y$ trece horas, y los niños de 6 a 12 años entre nueve y doce horas..$^{15}$ Los niños que cumplen con estos criterios tienen una mejor salud incluyendo: mejor atención, comportamiento, aprendizaje, memoria, calidad de vida, salud física y mental. Si la cantidad de horas es menor o mayor que la recomendada según edad puede generar riesgos, tales como: estar más propenso a accidentes, lesiones, hipertensión, obesidad, diabetes, y depresión. ${ }^{16}$
En relación con la calidad de sueño en pacientes pediátricos y adolescentes con BS, se ha observado mediante polisomnografía, una alta incidencia de micro-despertares. Estos episodios ocurren principalmente en la etapa dos de sueño no-REM y REM, se postula que el BS afectaría la calidad y la propiedad recuperativa del sueño, generando problemas de tipo conductual y atencional, influyendo negativamente en el desempeño escolar. Por otro lado, se postula que existe una asociación entre cantidad de horas de sueño y BS, señalando que niños que duermen menos de ocho horas por noche están más predispuestos a tener bruxismo. ${ }^{3}$

Con respecto, a los trastornos de sueño, se ha visto una prevalencia durante la infancia entre un 5 y un $40 \%$ de acuerdo con diferentes estudios. Esta amplia distribución de prevalencia se debe a que no es fácil ni existe un consenso para establecer la patología, además, estos desórdenes están influenciados según grupo etario preescolares, escolares y adolescentes. ${ }^{17}$

La Asociación Americana de Medicina del Sueño (AASM) publica en el año 2014 la tercera edición de la Clasificación Internacional de Trastornos del Sueño (ICSD-3). Ésta incluye seis divisiones clínicas principales: Insomnio, Trastornos respiratorios relacionados con el sueño, Trastornos centrales de la hipersomnolencia, Trastornos circadianos de sueño y vigilia, Parasomnias y Trastornos del movimiento relacionados con el sueño, cada uno desglosado en distintas patologías, con su propia clasificación, diagnóstico y codificación. ${ }^{4,28}$

En relación a la "Escala de alteraciones del sueño en la infancia" del Dr. O. Bruni et al. (en adelante, SDSC por su sigla en inglés "The Sleep Disturbance Scale for Children"), proporciona una medida estandarizada de las alteraciones de sueño en niños y adolescentes, define valores normales en la población, identifica niños que tienen el sueño perturbado y distingue los diferentes subconjuntos de elementos que se pueden usar como prueba de detección áreas específicas de los trastornos de sueño. ${ }^{15}$ Esta encuesta, fue originalmente validada en niños de 6 a 16 años, pero en el 2013 los mismos investigadores la aplicaron para preescolares, incluyendo a niños de 3 a 6 años, llegando a la conclusión que es una herramienta también válida en la edad preescolar. Sin embargo, al tener diferentes prevalencias propias de la edad de los distintos trastornos del sueño, las preguntas fueron agrupadas de forma diferente. Los trastornos que se desglosan de la SDSC para niños de 3 a 6 años son las siguientes: Parasom- 
nias, trastornos de inicio y mantenimiento, problemas respiratorios, excesiva somnolencia, hiperhidrosis de sueño y sueño no reparador. ${ }^{17}$

El propósito de este trabajo fue estudiar la relación entre el BS y dichas alteraciones de sueño en niños preescolares que asisten a la práctica habitual de la clínica Odontológica de la Universidad Mayor de Santiago, Chile: Diplomado de Odontología Pediátrica, Odontología Integral del Niño I y II.

\section{MATERIALES Y MÉTODO}

Se realizó estudio observacional, transversal de prevalencia. Se utilizó como variable independiente la presencia de bruxismo y como variable dependiente las alteraciones de sueño en niños. Se midió de acuerdo a los siguientes parámetros:

\section{BRUXISMO DE SUEÑO}

Considerando que la muestra estuvo constituida por niños. En este estudio se utilizó una versión modificada de los criterios diagnósticos según la Clasificación internacional de trastornos del sueño de la Academia Americana de Medicina del Sueño en su tercera versión. ${ }^{18}$

De acuerdo con ello, debían cumplirse el siguiente par de criterios:

A. Reporte de episodios regulares o frecuentes de apriete o rechinamiento dentario a tutores encargados.

B. La presencia de uno o más de los siguientes signos y síntomas clínicos:

-Presencia de bruxofacetas con exposición de dentina a nivel incisal u oclusal.

-Fractura de dientes o pérdida de restauraciones sin causa aparente.

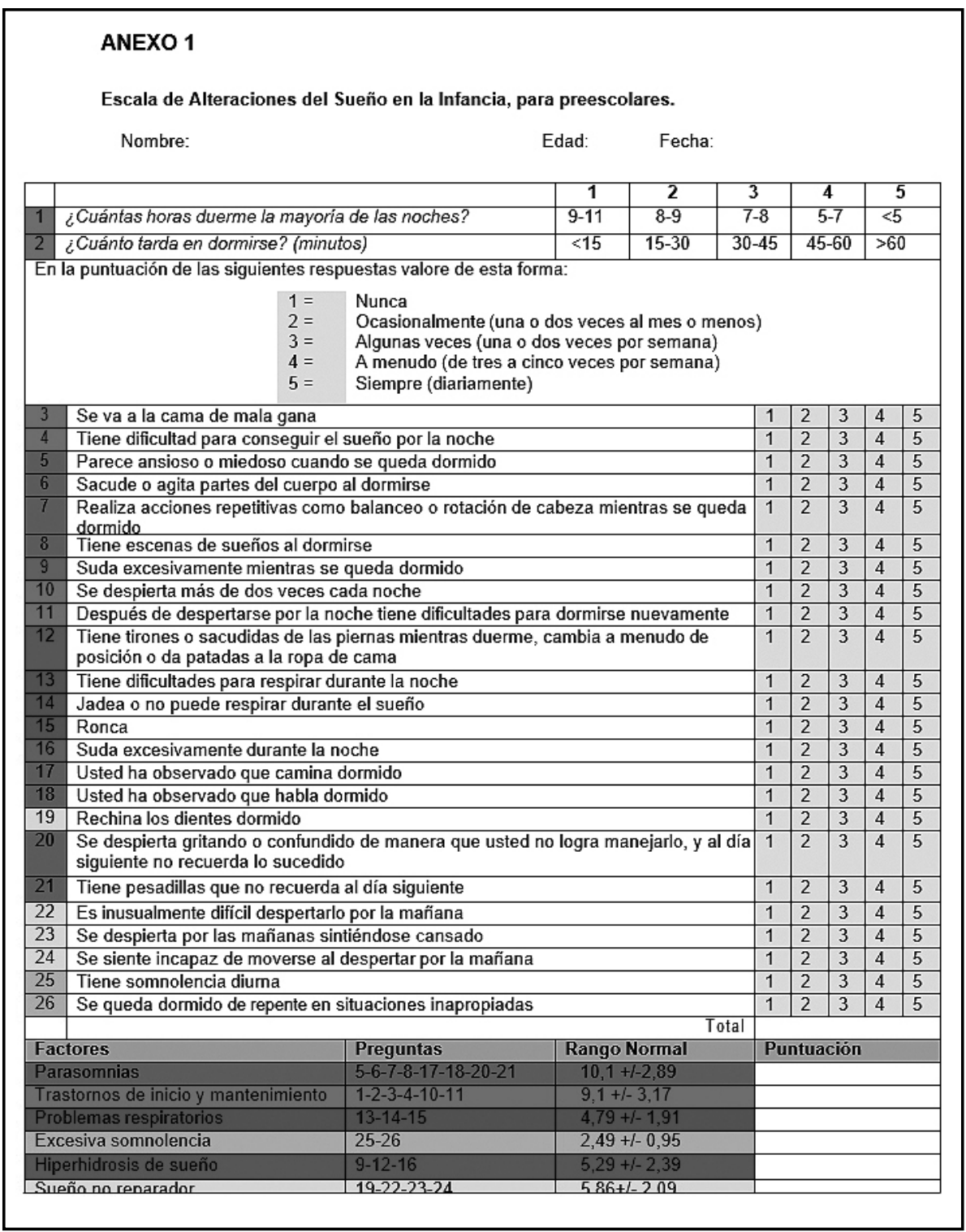

-Dolor a la palpación de la musculatura temporal o maseterina.

-Dolor o fatiga matutina transitoria de la musculatura mandibular.

-Dolor de cabeza temporal.

-Bloqueo de mandíbula al despertar (en acuerdo con informes anteriores de apriete dentario durante el sueño).

\section{TRAstornos DEL SUEÑo}

Se realizó la encuesta "Escala de alteraciones del sue- 
Köstner Uribe S, Brunet Echavarría J, Tapia Vargas A.

ño en la infancia" a los padres, un cuestionario que tiene por objetivo evaluar los trastornos de sueño en niños de edad preescolar, en los últimos seis meses. ${ }^{15}$

Se consideró una muestra de 100 niños que asistieron a la práctica habitual de la Clínica Odontológica de la Universidad Mayor: Diplomado de Odontología Pediátrica, Odontología Integral del Niño I y II, durante los meses de julio y agosto de 2017.

Se consideró como criterios de inclusión a niños y niñas de 3 a 6 años que tenían dentición temporal o mixta primera fase, pacientes en el cual el tutor aceptó estudio (consentimiento informado), contestó encuesta de SDSC (ANEXO 1) y ficha anamnésica para diagnóstico de bruxismo (ANEXO 2).

Se consideró como criterios de exclusión pacientes con tratamiento de ortodoncia interceptiva (previa o actual), pacientes que consumían medicamentos que alteren la actividad muscular (antidepresivos serotoninérgicos, neurolépticos, antidopaminérgicos $\mathrm{y} / \mathrm{o}$ anfetaminas para tratamiento de déficit atencional) y pacientes que rehusaron ser examinados.

Un investigador entrenado y calibrado realizó el examen clínico, mientras el otro se encargó de obtener el consentimiento informado y realizar las encuestas a los tutores. Lo anterior, con el fin de disminuir la posibilidad de sesgos durante la inspección clínica.

Se realizó calibración del examinador de la siguiente forma:

1. Para la palpación muscular, se utilizó balanza Ohaus ${ }^{\circledR}$ Precision Standard TS400D como método de calibración. El evaluador mantuvo por intervalos de 10 segundos 500 gramos de presión con los dedos pulgar, índice y medio de ambas manos.

2. Para el diagnóstico de bruxofacetas y para poder diferenciarlas de degaste fisiológico, el evaluador realizó calibración con 10 pacientes de la Clínica Odontológica de la Universidad Mayor, que posteriormente no participaron en el estudio. Apoyado por un especialista experto en Odontopediatría y otro en Oclusión, se llegó al consenso de bruxofacetas, considerando como patológica la presencia de islotes de dentina mayor a $1 \mathrm{~mm}$ (grado 3 o más según índice de desgaste dentario de Smith y Knight), tanto en molares, incisivos y caninos (Fig. 1). Se aplicó el test de concordancia inter-observador coeficiente de Kappa, en el cual hubo una concordancia de $\mathrm{K}=1 .^{29}$

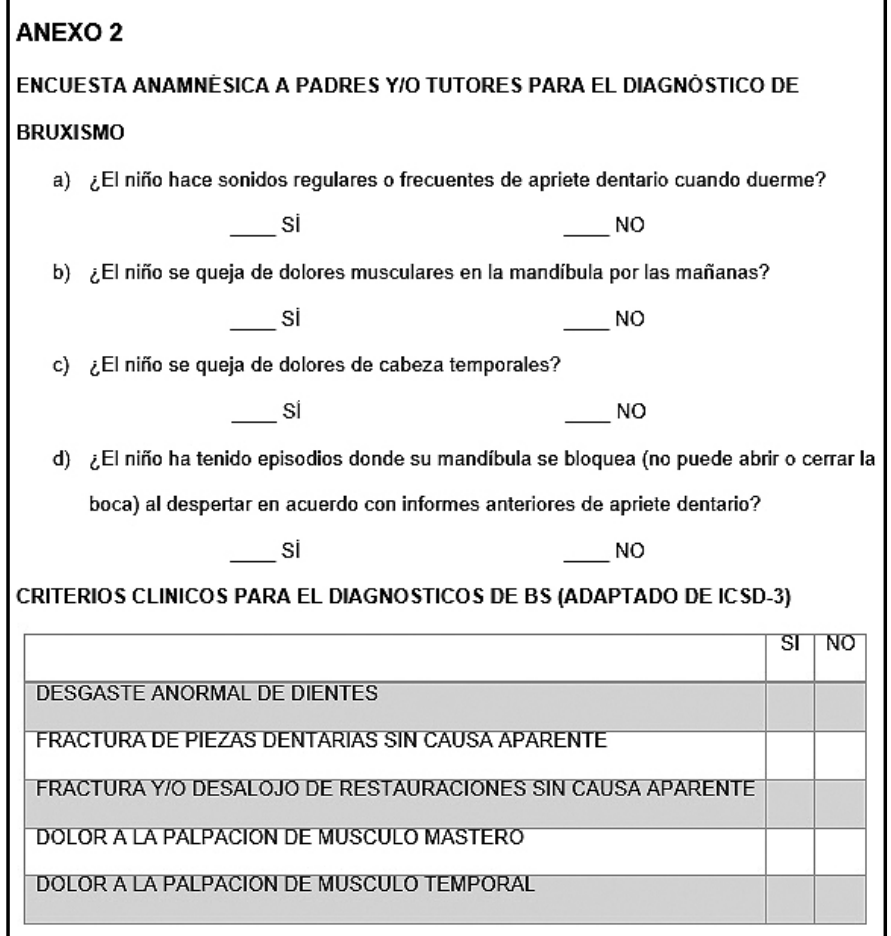

Para el análisis estadístico de los resultados, se utilizó el test Chi cuadrado y test de Regresión logística, que permitieron determinar si existían diferencias estadísticamente significativas cuando el $p$ value es $<0,05$. Ambos test se analizaron en software estadístico STATA® Versión 15.

\section{RESULTADOS}

Se evaluaron 100 niños, 51 mujeres y 49 hombres, de edades entre los 3 años y 6 años 11 meses. Los datos y resultados obtenidos se agruparon en tablas de frecuencia y gráficos de barras para su mejor comprensión. Se realizó tabulación para evaluar prevalencia de BS y prevalencia de trastornos de sueño (TS).

Se analizó mediante el test de proporciones Chi cuadrado, para determinar si existían diferencias estadísticamente significativas $(p<0,05)$ para determinar diferencia según género en pacientes con BS y la asociación entre trastornos de sueño y género.

Además, se aplicó Test de Regresión logística para determinar relación de BS con cada uno de los trastornos de sueño.

Con respecto a la prevalencia de BS, se observó que 47 pacientes presentaban la parafunción, de los cua- 
les un $53,19 \%$ correspondió a hombres y un $46,81 \%$ a mujeres (Fig. 2).

Según el Test Chi cuadrado, no existe diferencia signficativa en cuanto a distinción según género en pacientes que padecen $B S(P=0,430)$.

En relación con los trastornos de sueño en el total de los preescolares evaluados, se encontró una alta prevalencia de los Trastornos de Sueño, pero no se observó diferencia significativa en cuanto a la distinción por género para ninguno de los trastornos de sueño (Tabla 1).

Se realizó Test de Regresión logística para determinar la asociación entre BS y cada uno de los trastornos de sueño especificados en la SDSC, obteniéndose los siguientes resultados:

Los trastornos que presentaron asociación significativa fueron la Parasomnia, que arrojó un $\mathrm{P}=0,017$, Problemas Respiratorios que arrojó un $\mathrm{P}=0,046$ (muy cercano a no considerarse con asociación significativa), la Hiperhidrosis de Sueño, arrojando un $\mathrm{P}=0,000$ y Sueño no reparador también con un $P=0,000$, lo que indica que los pacientes que padecen de estos trastornos tienen una asociación positiva con la presencia de BS.

Entre estos trastornos, el Sueño no reparador, con un Odds ratio de 6,06, y la Hiperhidrosis de Sueño, con un Odds ratio de 3,84. fueron los que tuvieron una diferencia significativa más considerable, y por consiguiente son los que presentan una mayor probabilidad de asociarse con BS. (Fig. 3).

\section{DISCUSIÓN}

Se han realizado muchos estudios buscando establecer la prevalencia, etiología y la posibilidad de encontrar factores asociados al BS, pero no ha sido posible establecer un consenso., 5, 7, 10 Dentro de las dificultades para determinar el diagnóstico, y por lo tanto la prevalencia de BS, destaca el hecho de que los estudios publicados utilizan distintos métodos de investigación y distintos criterios clínicos que no permiten unificar los datos y por lo tanto no se obtienen resultados concluyentes. Además, no existen criterios diagnósticos para TTM ajustados para niños, por lo cual son utilizados aquellos validados en adultos, los que no necesariamente representan su realidad ni consideran en ellos su estado cognitivo (inmadurez). ${ }^{19}$

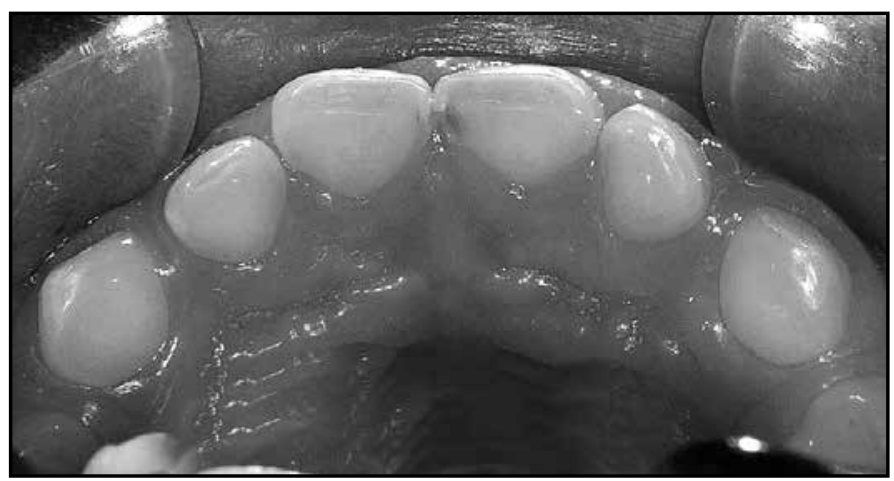

Fig. 1. Fotografía de desgaste patológico grado 3 en incisivos centrales superiores.

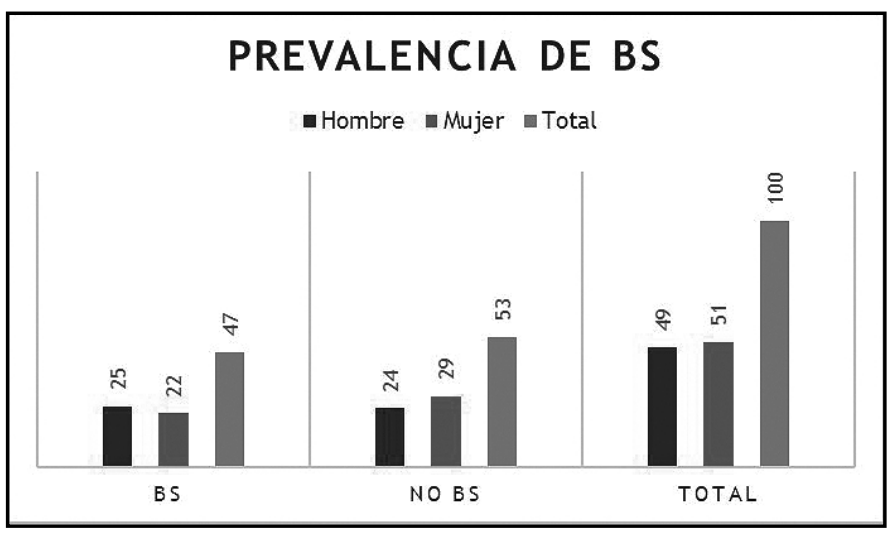

Fig. 2. Prevalencia de BS en pacientes preescolares atendidos en el Diplomado de Odontología Pediátrica y Odontología Integral del Niño I y II, de la Universidad Mayor.

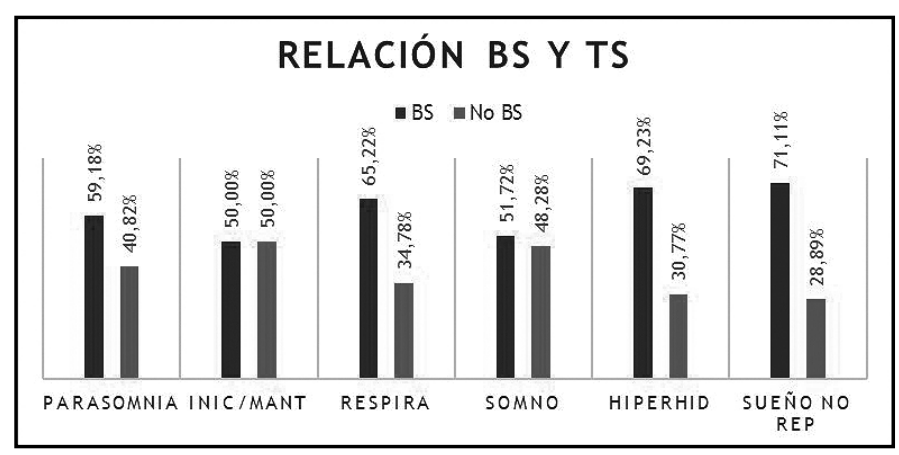

Fig. 3. Asociación de BS con cada TS de la SDSC.

Respecto del dolor, estudiar cualquier TTM en niños se ve dificultado por el hecho de que en general y dependiendo de la edad, no pueden discriminar bien la sensación dolorosa. Los niños pequeños confunden presión con dolor y no son capaces de cuantificar su malestar. ${ }^{19}$ Es por esto, que en este estudio se utilizó una pregunta con respuesta dicotómica por parte del paciente pediátrico (Sí/No), con el fin de disminuir las probabilidades de una respuesta vaga o dudosa. 
Köstner Uribe S, Brunet Echavarría J, Tapia Vargas A.

Trastornos de sueño asociados a bruxismo de sueño en niños entre 3 y 6 años de edad atendidos en la Clínica Odontológica de la Universidad Mayor de Santiago, Chile

La AASM determina como un criterio principal de diagnóstico de BS la presencia de sonidos regulares o frecuentes de apriete dentario que ocurran durante el sueño. ${ }^{4}$ Sin embargo, los parámetros 'regular o frecuente' no se describen en las guías diagnósticas, por lo que se consideró como 'frecuente' el reporte de episodios de apriete dentario más de una vez a la semana, tal como se estableció en el estudio de Stuginiski-Barbosa et al. ${ }^{18}$ Para el diagnóstico de los pacientes considerados en la muestra, se tuvo que consultar mediante encuesta a los padres o tutores por la ocurrencia de estos sonidos de apriete dentario.

Existe un amplio rango de prevalencia de BS en niños, la mayoría de los estudios epidemiológicos están basados en el auto-reporte, o el reporte de padres o tutores. ${ }^{5}$ En la investigación de Serra-Negra et al. se investigó la prevalencia de BS a través de una encuesta a los padres, en la cual se observó una prevalencia de BS de un 35,3\% en pacientes de alta vulnerabilidad social de Brasil.10 En el estudio de Manfredini et al., el grupo que mostraba mayor prevalencia estuvo constituido por niños de 3 a 5 años, con valores entre $8.5 \%$ y $25,1 \% .{ }^{20}$ En otro estudio en que sólo se utilizó el criterio de rechinamiento nocturno de la AASM, solamente un $14 \%$ tenía BS. ${ }^{21}$ Este menor porcentaje con respecto al resto de la literatura podría deberse a que no se consideró inspección clínica para el diagnóstico.

Al comparar todos estos datos con este estudio, se observa que es determinante el método diagnóstico que se utiliza, ya que no se asegura que, con la encuesta a padres que muchas veces desconocen la presencia del hábito parafuncional en sus hijos, se obtenga un diagnóstico correcto, por lo tanto, se debería considerar indudablemente el examen clínico de los pacientes por parte de un profesional.

Se ha analizado en diferentes estudios si existe diferencia significativa entre hombres y mujeres que padecen de bruxismo. En el estudio de Carvalho et al. se obtuvo una diferencia significativa entre hombres $\mathrm{y}$ mujeres, en que el porcentaje de las mujeres que tenían bruxismo era de un 53,8\%, en comparación a los hombres, los que presentaban un $46,2 \%$ de bruxismo. ${ }^{22}$ Sin embargo, en el estudio realizado por Simoes y Lopes en Sao Paulo, Brasil, en el cual se observó una prevalencia de un 55,3\% de niños con bruxismo reportado por los padres (sin clasificar el BS o de vigilia), también fue evaluada la relación de género y edad, sin encontrarse diferencia significativa. ${ }^{11}$ En relación al BS, en general se ha establecido que no existe diferencia en la presentación en hombres o mu-

\begin{tabular}{|c|c|c|c|}
\hline & HOMBRES & MUJERES & TOTAL \\
\hline PARASOMNIA & $\begin{array}{c}27 \\
(55,10 \%)\end{array}$ & $\begin{array}{c}22 \\
(44,90 \%)\end{array}$ & 49 \\
\hline INICIO Y MANTENIMIENTO & $\begin{array}{c}13 \\
(43,33 \%)\end{array}$ & $\begin{array}{c}17 \\
(56,67 \%)\end{array}$ & 30 \\
\hline PROBLEMAS RESPIRATORIOS & $\begin{array}{c}12 \\
(52,17 \%)\end{array}$ & $\begin{array}{c}11 \\
(47,83 \%)\end{array}$ & 23 \\
\hline EXCESIVA SOMNOLENCIA & $\begin{array}{c}17 \\
(58,62 \%)\end{array}$ & $\begin{array}{c}12 \\
(41,38 \%)\end{array}$ & 29 \\
\hline HIPERHIDROSIS DE SUEÑO & $\begin{array}{c}20 \\
(51,28 \%)\end{array}$ & $\begin{array}{c}19 \\
(48,72 \%)\end{array}$ & 39 \\
\hline SUEÑO NO REPARADOR & $\begin{array}{c}23 \\
(51,11 \%)\end{array}$ & $\begin{array}{c}22 \\
(48,89 \%)\end{array}$ & 45 \\
\hline
\end{tabular}

jeres, ${ }^{7,10,11,22}$ lo que coincide con esta investigación $(\mathrm{P}=0,430)$.

En relación a los trastornos de sueño, una gran cantidad de literatura ha sugerido que éstos son bastante comunes en niños y adolescentes. ${ }^{15,23,24,25}$ Sin embargo, la frecuencia de estos trastornos no ha sido claramente establecida, aunque se ha descrito que un 35 - 46\% de escolares tienen dificultades para dormir y un $14 \%$ de la población escolar tiene déficit de sueño ("poor sleepers") 15

Se ha reportado una asociación de un 29\% entre BS y trastornos del sueño y parasomnias. ${ }^{5}$ La mayoría de estas parasomnias (somniloquia, pesadillas, enuresis, sonambulismo, despertares confusos y terrores de sueño) son más prevalentes en niños que en adultos. Estudios han demostrado que los niños con estas parasomnias reportaron apriete dentario durante el sueño más frecuentemente que niños sin parasomnias. Es más, distintos estudios han indicado que estas parasomnias y el BS pueden compartir predisposiciones genéticas comunes, ${ }^{6}$ lo que concuerda con este estudio en el cual se estableció una relación positiva entre parasomnias y $\mathrm{BS}(\mathrm{P}=0,017)$.

El BS ha sido frecuentemente asociado a trastornos respiratorios, como roncar y AOS, se habla de una prevalencia de un 50\% en la población pediátrica, y se ha observado que después de realizar tonsilectomía o adenotonsilectomía, éste disminuye considerablemente. ${ }^{5}$ Una posible explicación de la asociación entre BS y AOS en niños, es que los infantes que rechinan sus dientes durante la noche (BS) lo hacen como resultado de una respuesta fisiológica para aumentar la permeabilidad de la vía aérea cuando tienen AOS. ${ }^{7}$ Sin embar- 
go, no se ha encontrado suficiente bibliografía para confirmarlo. Según el estudio realizado por Ferreira et al., en el cual se evaluaron niños preescolares de 3 a 6 años, sólo un 2,82\% presentó ambas condiciones (BS y AOS), pero no se encontró asociación estadística. ${ }^{7}$ No parece haber evidencia significativa para apoyar una relación de causa/efecto entre los trastornos respiratorios relacionados con el sueño y el BS. ${ }^{26}$ En este estudio sí se ha establecido una asociación estadística entre los problemas respiratorios y BS, pero con un p-value de 0,046, en el límite de no ser significativo. Por lo tanto, esta asociación tendría poca consistencia.

Con respecto a la hiperhidrosis de sueño, en el estudio de O. Bruni en preescolares se describe que los padres asociaron la sudoración nocturna a excesivos movimientos durante la noche. ${ }^{17}$ En concordancia con lo anterior, la AASM clasifica al BS como un trastorno de movimiento relacionado al sueño. ${ }^{4}$

El estudio de Kato et al., asocia los movimientos relacionados al sueño con el BS, presentando una prevalencia de un $10 \%$ de pacientes que padecen del síndrome de piernas inquietas y BS, aunque este trastorno es más común en la población adulta. ${ }^{6}$ Esto podría asociarse a que se puede generar un aumento de la temperatura corporal y por lo tanto una hiperhidrosis durante el sueño, que podría apoyar la asociación estadística considerable entre este trastorno y BS en la presente investigación $(P=0,000)$. Se debe tener en cuenta, que no se consideran factores ambientales como la temperatura de la habitación, la cantidad de ropa de cama, grosor del pijama o estación del año, por lo tanto, la evaluación de este parámetro es subjetiva.

Como conclusión de este estudio podemos afirmar que existe una relación entre BS y algunos trastornos de sueño, tales como Parasomnias, Problemas Respiratorios, Hiperhidrosis del Sueño y Sueño no Reparador.

Derivado de los concluido se destaca la importancia de la educación sobre la higiene de sueño en niños, definida como un conjunto de recomendaciones conductuales y ambientales, destinadas a promover el sueño saludable, ya que existen varias razones para considerar su potencial para promover la salud en la población en general. ${ }^{27}$
Por último, sería de interés a futuro el seguimiento y reevaluación de la muestra participante en esta investigación para verificar si la prevalencia de BS disminuye con los años como lo establece en la evidencia científica al respecto.

Financiamiento: Esta investigación no recibió financiamiento externo.

Conflicto de interés: Los autores declaran no tener conflictos de interés.

\section{BIBLIOGRAFIA}

1. LOBBEZOO F, AHLBERG J, GLAROS AG, KATO T, KOYANO K, LAVIGNE GJ, ET AL. Bruxism defined and graded: an international consensus. J Oral Rehabil. 2013 Jan; 40(1): 2-4.

2. FRUGONE R, RODRÍGUEZ C. Bruxismo. Av Odontoestomatol 2003 May-Jun;19 (03): 12330.

3. FIRMANI M, REYES M, BECERRA N, ET AL. Bruxismo de sueño en niños y adolescentes. Rev Chil Pediatr 2015; 86(05): 373-9.

4. Zucconi M, Ferri R. European Sleep Research Society, Sleep Medicine Textbook, Chapter B.1 Assessment of sleep disorders and diagnostic procedures, 2014.

5. Carra, M., Bruni, O., Huynh, N. Topical Review: Sleep Bruxism, Headaches, and Sleep-Disordered Breathing in Children and Adolescents. J Orofac Pain 2012;26: 267-76.

6. Kato T, Yamaguchi T, Okura K, et al. Sleep less and bite more: Sleep disorders associated with occlusal loads during sleep. J Prosthodont Res 2013;57: 69-81.

7. Ribeiro N, Fernandes dos Santo J, Bertolini M, et al. Sleep bruxism associated with obstructive sleep apnea syndrome in children. Cranio 2015;33(4):251-5.

8. Kato T, Thie N., Montplaisir J., et al. Bruxism and orofacial movements during sleep. Denl. Cl. Of NA 2001; 45(4): 657-84.

9. Serra Negra JM, Tirsa Costa D, Martins P. Evaluation of parents/guardian knowledge about 
Köstner Uribe S, Brunet Echavarría J, Tapia Vargas A.

Trastornos de sueño asociados a bruxismo de sueño en niños entre 3 y 6 años de edad atendidos en la Clínica Odontológica de la Universidad Mayor de Santiago, Chile

the bruxism of their children: Family knowledge of bruxism. J Indian Soc Pedod Prev Dent 2013;31(3): 153-8.

10. Serra-Negra J, Paiva S, Seabra A, et al. Prevalence of sleep bruxism in a group of Brazilian schoolchildren. Eur Arch Paediatr Dent 2010;11(4).

11. Simonoes-Zenari M, Lopes $M$. Factors associated to bruxism in children from 4-6 years Pro Fono 2010;22(4):465-72.

12. Guevara Gómez, S; Ongay Sánchez, E; Castellanos, J. Avances y limitaciones en el tratamiento del paciente con bruxismo. Revista ADM 2015; 72 (2): 106-14.

13. Norman R. Friedman. Polysomnography Should Not Be Required Both Before and After Adenotonsillectomy for Childhood Sleep Disordered Breathing. J Clin Sleep Med. 2007 Dec 15; 3(7): 678-80.

14. Loomans B, Opdam N, Attin T et al. Severe Tooth Wear: European Consensus Statementon Management Guidelines. J Adhes Dent 2017 19:111-9.

15. Bruni O., Ottaviano S, Guidetti V, et al. The Sleep Disturbance Scale for Children (SDSC), Construction and validation of an instrument to evaluate sleep disturbances in childhood and adolescence. J. Sleep Res 1996;5: 251-61.

16. Paruthi S, Brooks LJ, D'Ambrosio C, et al. Recommended Amount of Sleep for Pediatric Populations: Consensus Statement of the American Academy of Sleep Medicine. J Clin Sleep Med 2016;12(6):785-6.

17. Romeo D, Bruni O, Brogna C et al. Application of the Sleep Disturbance Scale for Children (SDSC) in preschool age. Eur J Paediatr Neurol 2013;17: 374-82.

18. Stuginski-Barbosa J, Costa J, Porporatti A et al. Agreement of the International Classification of Sleep Disorders Criteria with polysomnography for sleep bruxism diagnosis: A preliminary study. J Prosthet Dent 2017;117(1): 61-6.

19. Romo F, Díaz W, Schulz R et al. Tópicos de Odontología Integral: Bruxismo Gráfica Metro- politana 2011: 273-87.

20. Manfredini D, Restrepo C, Diaz-Serrano K, et al. Prevalence of Sleep Bruxism in Children: A systematic review of the literature. J Oral Rehabil 2013;40: 631-42.

21. Vierira R, Gonzaga G, Drumond CL et al. Prevalence of Sleep Bruxism and Associated Factors in Preschool Children. Pediatr Dent 2014;36(1):46-50.

22. Brito A, Moura M, Nunes da Silva J et al. Bruxism and quality of life in schoolchildren aged 11 to 14 . Cien Saude Colet 2015;20(11): 3385-93.

23. Carter K, Hathaway $\mathrm{N}$ y Lettieri C. Common sleep disorders in children. Am Fam Physician 2014;89: 368-77.

24. Licis A. Sleep disorders: Assessment and treatment in preschool-aged children. Child Adolesc Psychiatr Clin N Am 2017;26(3):587-95.

25. Moturi S y Avis K. Assessment and treatment of common pediatric sleep disorders. Psychiatry (Edgmont) 2010; 7(6):24-37.

26. Bender S. Sleep bruxism and sleep-disordered breathing. J Esthet Restor Dent 2016;28: 67-71.

27. Irish L, Kline $\mathrm{C}$, Gunn $\mathrm{H}$ et al. The role of sleep hygiene In promoting public health: a review of empirical evidence. Sleep Med Rev 2015;22: 23-6.

28. American Academy of Sleep Medicine. International classification of sleep disorders, revised: Diagnostic and coding manual. Chicago, Illinois: American Academy of Sleep Medicine, 2001.

29. Cerda J, Villarroel L. Evaluación de la concordancia inter-observador en investigación pediátrica: Coeficiente de Kappa. Rev Chil Pediatr 2008;79(1): 54-8.

\section{AUTOR DE CORRESPONDENCIA:}

Sofía Magdalena Köstner Uribe.

Teléfono +56 932692343 .

Dirección: Av. Presidente Kennedy 7120, oficina 205,

Vitacura, Región Metropolitana, Chile.

Correo: sofiakostner@gmail.com. 